\title{
Ribosomal internal transcribed spacer sequences are identical among Mycobacterium avium-intracellulare complex isolates from AIDS patients, but vary among isolates from elderly pulmonary disease patients
}

\author{
Koen A. L. De Smet, ${ }^{1}+$ Ivor N. Brown, ${ }^{2}$ Malcolm Yates ${ }^{3}$ and Juraj Ivanyi ${ }^{1}$
}

Author for correspondence: Koen De Smet. Tel: +44 1717231252 ext. 5746. Fax: + 441712626299.
e-mail: k.desmet (Qic.ac.uk

1 MRC Tuberculosis and Related Infections Unit, Clinical Sciences Centre, Hammersmith Hospital, DuCane Road, London W12 ONN, UK

2 Department of Medical Microbiology, St Mary's Hospital Medical School, Norfolk Place, London W2 1PG, UK

3 Public Health Laboratory Service, Regional Tuberculosis Centre, Dulwich Hospital, London SE22 8QF, UK

\begin{abstract}
Sequencing 280 bp of the internal transcribed spacer (ITS) between the 165 and 235 rRNA genes in a collection of 46 clinical isolates of the Mycobacterium avium-intracellulare complex (MAl complex) identified nine different sequences, grouping these isolates in nine 'ITS sequevars'. This analysis extends the subdivision within the MAl complex to 18 ITS sequevars and also improves discrimination from other mycobacterial species. Evaluation of the sequevar grouping among different clinical sources revealed strong association of the $\boldsymbol{M}$. avium sequevar Mav-B with AIDS and with lymphadenitis in children (18 out of 20 and 3 out of 3 respectively). Isolates from elderly patients with pulmonary disease and not suspected of being HIV infected belonged predominantly to $M$. intracellulare ITS sequevars and sequevars not assigned to either $M$. avium or $M$. intracellulare. On the other hand, animal isolates were of both the Mav-A and Mav-B sequevars. We conclude that ITS sequevar typing is an accurate way of identifying distinct MAI complex strains. The observed differences between clinical sources suggest that ITS sequevars reflect possibly important, biologically and clinically relevant polymorphisms between MAl complex organisms.
\end{abstract}

Keywords: $M$. avium-intracellulare complex, DNA sequence analysis, $16 \mathrm{~S}$ ribosomal RNA, AIDS, virulence

\section{INTRODUCTION}

Mycobacterium avium-intracellulare complex (MAI complex) organisms cause chronic pulmonary infections in elderly people with pre-existing lung disease, lymphadenitis in children and systemic bacterial infection in patients with AIDS in the developed world (Rosenzweig \& Scheulter, 1981; Lai et al., 1984; Inderlied et al., 1993). M. avium also causes tuberculosis in birds and mainly local granulomatous infections in cattle and other animal species (Thoen et al., 1994). The possible association between these diverse clinical manifestations with as yet unknown virulence markers, possibly occurring in only certain subtypes of the MAI complex, represents an important

†Present address: Department of Medical Microbiology, St Mary's Hospital Medical School, Norfolk Place, London W2 1PG, UK.

Abbreviations: MAl, M. avium-intracellulare; ITS, internal transcribed spacer. subject for research. However, differentiation of the species and subspecies within the MAI complex remains difficult, despite existing methods of serotyping, DNA hybridization or DNA sequencing (Saito et al., 1989, 1990; Thorel et al., 1990; Tsang et al., 1992; Kirschner et al., 1993). Therefore, clinical identification is often limited to assigning an isolate merely to the MAI complex (Inderlied et al., 1993).

The MAI complex includes at least two genetically different species, $M$. avium and $M$. intracellulare. $M$. avium contains three subspecies, $M$. avium subsp. avium, $M$. avium subsp. paratuberculosis, which causes Johne's disease in cattle, and $M$. avium subsp. silvaticum, the 'wood pigeon bacillus' (Thorel et al., 1990).

Serotyping divides the MAI complex into 28 serovars. Reference strains with serovars $1-6,8-11,21$ and 28 are considered to be $M$. avium, while those with serovars 7, 12-20 and 25 are assigned to $M$. intracellulare (Saito et al., 
1989; Böddinghaus et al., 1990; Tomioka et al., 1993). Serotyping showed that patients with AIDS are infected almost exclusively with $M$. avium (Tsang et al., 1992). However, up to one-third of all isolates cannot be serotyped due to autoagglutination or failure to react with any serum (Tsang et al., 1992; De Smet et al., 1995) and inconsistencies between serotyping of the same strains in six different laboratories have been reported (Wayne $e t$ al., 1993).

A kit based on the hybridization with species-specific oligonucleotides (Accuprobe, from Gen-Probe) can differentiate between $M$. avium and $M$. intracellulare at a genetic level. Using this kit, it has been demonstrated that these two species have different drug resistance profiles (Guthertz et al., 1989; Saito et al., 1989) and that the prognosis of pulmonary disease is better for patients with $M$. intracellulare infection (Yamori \& Tsukamura, 1992). However, a number of MAI complex reference strains as well as clinical isolates have been found negative with both Accuprobes (De Smet et al., 1995; Saito et al., 1990, Tomioka et al., 1993; Wayne et al., 1993).

Sequencing part of the $16 \mathrm{~S}$ rRNA gene has also been used for identifying mycobacteria, including those from the MAI complex (Kirschner et al., 1993). This supported the classification based on serotyping and found sequence variation within $M$. intracellulare, but not in $M$. avium (Böddinghaus et al., 1990).

Recently, subtyping of MAI complex isolates has been performed by sequencing the $16 \mathrm{~S}-23 \mathrm{~S}$ rDNA internal transcribed spacer (ITS) (Frothingham \& Wilson, 1993). Because this $280 \mathrm{bp}$ region of the rRNA operon is transcribed but does not code for a final product, it allows more sequence variation than the $16 \mathrm{~S} \mathrm{rDNA}$, and is therefore suitable for sub-species classification (Frothingham \& Wilson, 1993). By analogy with the definition for 'serovar', a strain with a specific ITS sequence is said to belong to a specific 'sequevar' (Frothingham \& Wilson, 1993). Analysis of 35 MAI complex reference strains identified four $M$. avium sequevars (named Mav-A to Mav-D), differing by one or two basepairs, while only one $M$. intracellulare sequevar (Min-A) was identified. Seven serovar reference strains belonged to sequevars with ITS sequences that differed from both Mav and Min ITS sequences by $10-31$ basepairs and were designated as MAC-A to MAC-G. These data support the view that the MAI complex consists of more than two species (Frothingham \& Wilson, 1993).

Sequevar typing has so far been applied only to a limited number of clinical isolates (Frothingham \& Wilson, 1994), but eight isolates from US patients with AIDS had the Mav-B sequevar and three the Mav-A sequevar. By contrast, MAI complex isolates from eight patients with pulmonary disease had MAC-A and Min-A sequevars.

The purpose of this study was to further evaluate sequevar typing using a collection of 46 MAI complex isolates from AIDS patients, pulmonary infections and lymphadenitis, and from animals. This analysis extends the number of different sequevars found in clinical samples from five to ten. The Mav-B sequevar was associated with AIDS and child lymphadenitis, whereas isolates with Mav, Min and MAC sequevars were found in elderly patients with pulmonary disease. Animals had only Mav sequevars, mainly Mav-A.

\section{METHODS}

Source of MAI complex isolates Forty-six isolates from several clinical sources were selected from a larger, previously described collection (Hellyer et al., 1991, 1993; De Smet et al., 1995). Selection was biased towards isolates that were serotyped and we avoided repeat isolates from the same patient. All isolates were identified biochemically as belonging to the MAI complex by a diagnostic laboratory. They have all been analysed for the presence of plasmids (Hellyer et al., 1993), all except the $M$. avium subsp. paratuberculosis isolates have been serotyped, and 28 of the 46 isolates were tested with the Accuprobe kit (De Smet et al., 1995).

Seven isolates were obtained from AIDS patients attending St Mary's Hospital, London, three US AIDS isolates were provided by Dr R. A. Ollar, St Vincent's Hospital, New York, USA, and the remaining AIDS isolates and all non-AIDS isolates were obtained from two diagnostic laboratories serving several hospitals in England and Wales. The organisms from 22 AIDS patients were isolated from faeces (11), sputum (5), blood (3) or other samples (3). Isolates from 12 elderly UK patients with pulmonary disease, not suspected of being HIV infected, were from sputum samples (six male and six female, aged 59-79 years). Four UK children with lymphadenitis provided cervical or submandibulary lymph node biopsy samples.

Of the animal MAI complex isolates, seven were isolated from cattle (including two $M$. avium subsp. paratuberculosis) and two each from pigs and pigeons. Animal isolates were provided by Dr A. Nolan of the Central Veterinary Laboratory, Weybridge, UK.

Extraction of DNA. The isolates were cultured on $\mathrm{LJ}$ slopes and DNA was extracted using $N$-cetyl- $N, N, N$-trimethylammonium bromide (CTAB) as described by Van Soolingen $e t$ al. (1991). The concentration was estimated by running an aliquot on a $0.8 \%$ agarose gel and diluted to approximately $10 \mathrm{ng} \mu \mathrm{l}^{-1}$.

Sequevar determination. The primers ' $16 \mathrm{~S}$ ' and ' $23 \mathrm{~S}$ ' were adapted from those described by Frothingham \& Wilson (1993), and had the sequences TTGTACACACCGCCCGTCA and CGATGCCAAGGCATCCACC. They were derived from the sequence near the ends of the $16 \mathrm{~S}$ and $23 \mathrm{~S}$ rDNA, and amplify a region of approximately $480 \mathrm{bp}$, containing the entire ITS sequence and the ends of both genes. Approximately $10 \mathrm{ng}$ genomic DNA was amplified in a total volume of $50 \mu \mathrm{l}$ containing $0.5 \mu \mathrm{M}$ of each primer, $200 \mu \mathrm{M}$ of each nucleotide, $5 \mu \mathrm{l} 10 \times$ Boehringer PCR buffer and $2.5 \mathrm{U}$ Taq polymerase (Boehringer), covered by one drop of mineral oil. The DNA was amplified with two cycles of $95^{\circ} \mathrm{C}$ for $2 \mathrm{~min}, 55^{\circ} \mathrm{C}$ for $1 \mathrm{~min}$ and $72^{\circ} \mathrm{C}$ for $1 \mathrm{~min}$, followed by 28 cycles of $92^{\circ} \mathrm{C}$ for $1 \mathrm{~min}, 55^{\circ} \mathrm{C}$ for $1 \mathrm{~min}$ and $72^{\circ} \mathrm{C}$ for $1 \mathrm{~min}$, and one cycle of $72^{\circ} \mathrm{C}$ for $9 \mathrm{~min}$, using an Omnigene (Hybaid). Blank controls (samples without DNA) were used for every five samples.

Five microlitres of this PCR reaction was analysed on a $1.5 \%$ $(\mathrm{w} / \mathrm{v})$ agarose gel. The PCR product was precipitated by mixing the remaining $45 \mu \mathrm{l}$ with $55 \mu \mathrm{l}$ of $25 \% \mathrm{PEG}, 6 \mathrm{mM} \mathrm{MgCl}$, $0.55 \mathrm{M}$ sodium acetate $\mathrm{pH} 5 \cdot 2$. This was left at room temperature for $15 \mathrm{~min}$, centrifuged, washed with $70 \%$ (v/v) ethanol and 
dissolved in $20 \mu \mathrm{l}$ water. Two microlitres was used for cycle sequencing with the Cyclist $\mathrm{Exo}^{-} \mathrm{Pfu}$ DNA sequencing kit (Stratagene), according to the manufacturer's instructions using the $23 \mathrm{~S}$ primer and the thermal cycling programme described above.

Sequencing part of the 165 ribosomal RNA gene. The primers '264' (sequence TGCACACAGGCCACAAGGGA) and '285' (GAGAG'T'T'TGATCCTGGCTCAG) are described by Kirschner et al. (1993) and amplify the 16S rRNA gene from nucleotide 9 to 1046 (numbering based on Kirschner et al., 1993). The reaction volume and composition were as described above and the DNA was amplified with 30 cycles of $94^{\circ} \mathrm{C}$ for $1 \mathrm{~min}, 68^{\circ} \mathrm{C}$ for $3 \mathrm{~min}$. The PCR product was precipitated and used for cycle sequencing as described above using the primer '244' (CCCACTGCTGCCTCCCGT) and the thermal cycling programme for the PCR with the primers 264 and 285. Species assignation was based on finding a perfect match with the DNA sequence of the hypervariable region $\mathrm{A}$, between nucleotides 129 and 266 (Kirschner et al. 1993).

Avoiding PCR contamination. To minimize the chance of contaminating pre-PCR samples with post-PCR products (the most common cause of false positives), a three-room strategy was used, with pre-PCR manipulations in one room, the amplification in a second and analysis and sequencing in a third (Kwok \& Higuchi, 1989). Since the sequencing itself amplifies only part of one strand and is thus a linear rather than an exponential amplification (producing only 30 partial copies of ssDNA rather than $>10^{9}$ copies of dsDNA), the production of significant amounts of false positives was minimal and all the sequencing manipulations were therefore performed in the third room.

Serotyping and Accuprobe analysis. The serotyping and Accuprobe analysis of these isolates has been described before (De Smet et al., 1995). Briefly, serotyping was performed by agglutination and adsorption according to Good \& Beam (1984), except that half the volumes were used throughout. Analysis with the Accuprobe culture identification kit (GenProbe) was according to manufacturer's instructions.

Phylogenetic analysis. Published sequevar sequences were obtained from the GenBank or EMBL databases: accession numbers L07855 (Mav-A); L07856 (Mav-B); L07857 (Mav-C); L07858 (Mav-D); L07859 (Min-A); L07847 (MAC-A); L07848 (MAC-B); L07849 (MAC-C); L07851 (MAC-D); L07852 (MAC-E); L07853 (MAC-F); L15620 (MAC-H); L15622 (MscA); L07854 (Mcs-B); L15623 (Mtb-A); X74495 (Mptb); X74056 (Mhab). All computer analyses were performed using the Wisconsin Sequence Analysis Package (GCG), version 8.0. Both GenBank and EMBL were searched for homologies using FASTA and BLASTN. Sequences were aligned using the PILEUP program, except for MTC-A and Mtb-A, which were edited before the alignment by adding some extra gaps. Default values of the program were used. Homologies between the aligned sequences were calculated using the program DISTANCES and the Jukes-Cantor correction method. The output of the DISTANCES program was used to construct a rooted phylogenetic tree with the GROWTREE program, using the UPGMA algorithm.

\section{RESULTS}

\section{Sequevar determination}

A PCR product for sequevar determination was obtained for all isolates at the first attempt. None of the blank controls gave false-positive amplification. Analysis of the
PCR products on a $1.5 \%$ agarose gel showed a single band of about $480 \mathrm{bp}$, except for isolate C8557, which appeared to be slightly smaller. Sequencing of the 45 different PCR products yielded one of 12 different sequences (Fig. 1). Of these, five corresponded to the previously described Mav-A, Mav-B, Min-A and MAC-A sequevars and the $M$. scrofulaceum sequevar Msc-A (Frothingham \& Wilson, 1993, 1994). Of the seven newly identified sequences, four differed by one to three basepairs from known Mav or Min sequevar sequences and we thus named the corresponding new sequevars Mav-E, Min-B, Min-C and Min-D (Fig. 1). Three new sequences differed by at least 15 basepairs (5\%) from the known ITS sequences and the corresponding sequevars were named MAC-I, Msi-A and MTC-A. The new sequevar sequences MAC-I, Mav-E, Min-B, Min-C, MinD, Msi-A and MTC-A were submitted to the EMBL database and assigned accession numbers Z46421 to Z46427 respectively.

\section{Mav and Min sequevars}

The three $M$. avium sequevar sequences Mav-A, Mav-B and Mav-E differed by one or two substitutions and one insertion. The vast majority of human isolates (22 out of 25) belonged to sequevar Mav-B, with only two Mav-A and one Mav-E. Mav-A was found in three out of five animal $M$. avium isolates (see Table 4). The two pig isolates were both Mav-B, while the cow and woodpigeon isolates were all Mav-A. Interestingly, the two $M$. avium subsp. paratuberculosis isolates belonged to the Mav-A sequevar. No isolate belonged to the previously reported Mav-C or Mav-D sequevars (Frothingham \& Wilson, 1993).

Four Min sequevar sequences differing by one to three substitutions were identified among the clinical isolates from pulmonary disease, but not from AIDS patients or animals (Fig. 1, Table 4).

\section{Other sequevars}

Five other sequences (in six isolates) differing by at least $15 \mathrm{bp}$ from $\mathrm{Mav}$ and Min sequevar sequences were identified (Fig. 1). Two of these had previously been designated as MAC-A and the $M$. scrofulaceum sequevar Msc-A (Frothingham \& Wilson, 1993, 1994). Three other sequences, identifying the sequevars MAC-I, Msi-A and M'TC-A, could not be assigned to known ITS sequences by searching the EMBL or GenBank databases.

These six isolates were further examined for species identification by sequencing part of their 16S rRNA gene (Table 1) (Kirschner et al., 1993). Isolate D32016 (sequevar MAC-A) had a region A $16 \mathrm{~S}$ rDNA sequence identical to $M$. intracellulare, and was thus identified as $M$. intracellulare. Isolates D31787 and D40469 were members of the same sequevar; their region A $16 \mathrm{~S}$ rDNA sequence differed slightly from the type strain of $M$. intracellulare, but was identical to the serotyping reference strain ' $M$. intracellulare serovar 18' (Böddinghaus et al., 1990). These isolates were thus also considered to belong to the MAI 

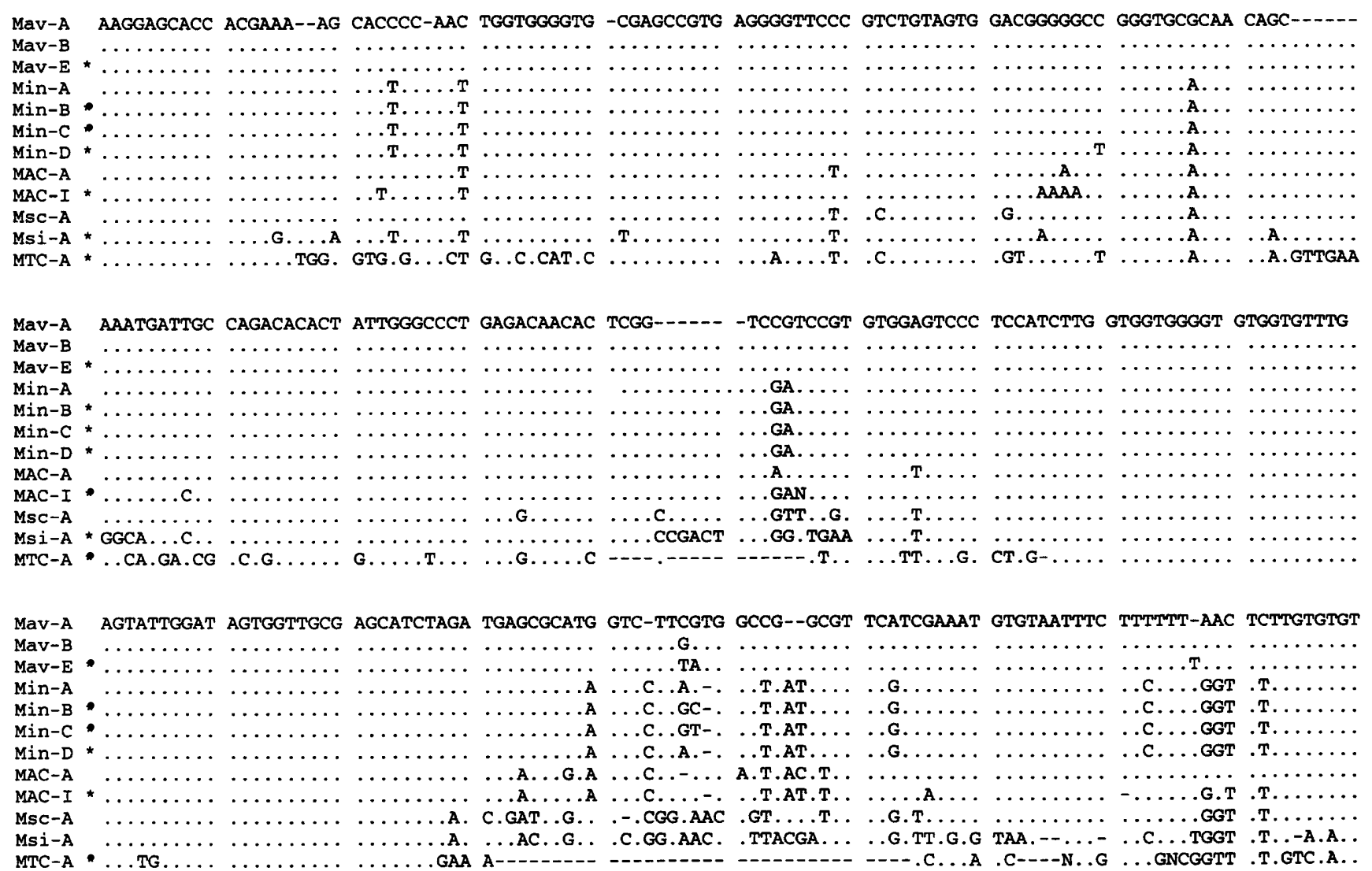

Fig. 1. Alignment of ITS sequences obtained from clinical isolates. Asterisks indicate newly defined sequevars.

Table 1. Alignment of region A of the $16 \mathrm{~S}$ rDNA sequences obtained from six clinical isolates

\begin{tabular}{|c|c|c|c|c|c|}
\hline \multirow[t]{2}{*}{ Isolate } & \multicolumn{3}{|c|}{ Nucleotide sequences* } & \multirow[t]{2}{*}{ Species } & \multirow{2}{*}{$\begin{array}{l}\text { ITS } \\
\text { sequevar }\end{array}$} \\
\hline & $129-159$ & $184-223$ & $250-266$ & & \\
\hline M. avium $\dagger$ & CAATCTECCCTGCACTTCGGGATAACCCTG & GACCTCAAGACGCATGTCTKCTEGTGGMAGCTHHT--GC & THETTEETEGGGTAACG & M. avium & \\
\hline D30216 & (.......................... & 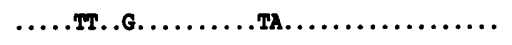 & ............ & $M$. intracellulare & MAC-A \\
\hline D31787 & ................... & 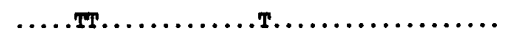 & ............. & MAI complex ser. 18 & MAC-I $\ddagger$ \\
\hline D40469 & n.......................... & 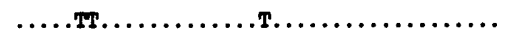 & .............. & MAI complex ser. 18 & MAC-I \\
\hline D13383 & т......................... & 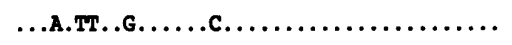 & $\ldots \ldots \ldots \ldots \ldots \ldots$ & M. simiae & Msi-A $\ddagger$ \\
\hline D40179 & & ..A.Tr..G.....c................... & .A.......... & M. scrofulaceum & Msc-A \\
\hline $\mathrm{C} 8557$ & TG....c....c................ & ......T.CTC....GTG.G.............TH. & …........ & M. terrae complex & MTC-A $\ddagger$ \\
\hline
\end{tabular}

* Only the regions used for species identification are shown.

$\dagger$ Sequence from Kirschner et al. (1993).

$\ddagger$ New sequevars.

complex. Consequently, the new ITS sequevar containing these two isolates was named MAC-I

The isolate D40179 (sequevar Msc-A) was confirmed by region A $16 \mathrm{~S}$ rDNA sequencing as $M$. scrofulaceum. Isolate C13383 was identified as $M$. simiae by region A 16 S rDNA sequencing, and the corresponding sequevar was thus named Msi-A. The region A $16 \mathrm{~S}$ rDNA sequence of isolate $\mathrm{C} 8557$ was not present in the EMBL or GenBank databases, but showed the highest homology to $M$. nonchromogenicum and $M$. triviale. It was therefore considered to be a member of the $M$. terrae complex, which contains these species (Wayne \& Kubica, 1986) and its sequevar thus named MTC-A.

All isolates tested had been included in this study because they had been identified biochemically as belonging to the MAI complex in a routine diagnostic setting. However, 
Table 2. Comparison of serovars with ITS sequevars

\begin{tabular}{|c|c|c|c|c|c|}
\hline & \multirow[t]{2}{*}{ Serovar } & \multicolumn{4}{|c|}{ No. of isolates with sequevar } \\
\hline & & Mav & Min & MAC & Other \\
\hline \multirow{9}{*}{$\begin{array}{l}\text { M. avium } \\
\text { associated serovars }\end{array}$} & 1 & 2 & - & - & - \\
\hline & 2 & 2 & - & - & - \\
\hline & 3 & 2 & - & - & - \\
\hline & 4 & 6 & - & - & - \\
\hline & 6 & 1 & - & - & - \\
\hline & 8 & 10 & - & - & - \\
\hline & 9 & 2 & - & - & - \\
\hline & 10 & 1 & - & - & - \\
\hline & 28 & 2 & - & - & - \\
\hline \multirow{5}{*}{$\begin{array}{l}\text { M. intracellulare } \\
\text { associated serovars }\end{array}$} & 12 & 1 & - & - & - \\
\hline & 13 & 1 & - & - & - \\
\hline & 18 & - & 1 & - & - \\
\hline & 19 & - & 1 & 1 & $1 \mathrm{Msi}-\mathrm{A}$ \\
\hline & 25 & - & - & 2 & - \\
\hline \multirow[t]{2}{*}{ Other serovars } & $12 / 28$ & - & 1 & - & $1 \mathrm{M}$ TC-A \\
\hline & Not typable & - & 4 & 1 & $1 \mathrm{Msc}-\mathrm{A}$ \\
\hline Total & & 30 & 7 & 4 & 3 \\
\hline
\end{tabular}

Table 3. Comparison of Accuprobe analysis with ITS sequevars

\begin{tabular}{|lcccc|}
\hline \multirow{1}{*}{ Accuprobe } & \multicolumn{4}{c|}{ No. of isolates with sequevar } \\
\cline { 2 - 5 } & Mav & Min & MAC & Other \\
\hline M. avium & 19 & - & - & - \\
M. intracellulare & - & 5 & 1 & - \\
Neither & - & - & - & 2 \\
Total & 19 & 5 & 1 & 2 \\
\hline
\end{tabular}

DNA sequencing showed that three of the 46 isolates were other mycobacterial species and had thus been incorrectly identified as MAI.

\section{Association of sequevars with serovar and Accuprobe analysis}

Most of the isolates had previously been serotyped and/or tested by Accuprobe (De Smet et al., 1995). All but two of the isolates with a Mav sequevar belonged to a serovar associated with $M$. avium (that is, serovars 1-6, 8-11, 21 or 28)(Table 2). The two aberrant isolates belonged to sequevar Mav-B and serovars 12 and 13, and were from a lymphadenitis and an AIDS patient respectively. There was no association of specific serovars with sequevars: different isolates with the same serovar could belong to either the Mav-A, Mav-B or Mav-E sequevar (data not shown). All the isolates belonging to the Min, MAC-I,
Msi-A and MTC-A sequevars were members of serovars associated with $M$. intracellulare, while the Msc-A and MAC-A isolates were not typable.

There was a perfect correlation between sequevar and Accuprobe analysis for identification of $M$. avium and $M$. intracellulare (Table 3). One isolate with the MAC-A sequevar was also positive with the $M$. intracellulare Accuprobe. This is in agreement with the region A $16 \mathrm{~S}$ DNA sequencing, which found an $M$. intracellulare sequence for this isolate. The other MAC-A isolate and the MAC-I isolates were not tested with the Accuprobe.

\section{Association of sequevars with the clinical source}

The four groups of isolates (AIDS, pulmonary disease, lymphadenitis and animals) showed clear differences in the distribution of sequevars (Table 4). Isolates from AIDS patients showed a striking predominance of sequevar Mav-B (18 out of 22 isolates, or 18 out of 20 MAI complex isolates) and one each of Mav-A, Mav-E, Msi-A and MTC-A. Interestingly, Mav-B was also found in three out of four lymphadenitis patients. By contrast, the Min-A sequevar was the most prevalent in pulmonary disease patients ( 4 out of 13 tested), but there was much more variety in this group. All Min and MAC sequevars identified were found only in this group and none in either AIDS, lymphadenitis or animals.

Three isolates were identified as other species, despite having been classified as MAI by biochemical means. $M$. scrofulaceum was isolated from a child with lymphadenitis, while the $M$. simiae and $M$. terrae isolates were from AIDS 
Table 4. Distribution of ITS sequevars among isolates from different sources

\begin{tabular}{|lrccc|}
\hline ITS & \multicolumn{4}{c|}{ No. of isolates from: } \\
\cline { 2 - 5 } sequevar & AIDS & $\begin{array}{c}\text { Pulmonary } \\
\text { disease }\end{array}$ & $\begin{array}{c}\text { Lymph- } \\
\text { adenitis }\end{array}$ & Animals \\
\hline Mav-A & 1 & 1 & - & $5^{*}$ \\
Mav-B & 18 & 1 & 3 & 2 \\
Mav-E & 1 & - & - & - \\
Min-A & - & 4 & - & - \\
Min-B & - & 1 & - & - \\
Min-C & - & 1 & - & - \\
Min-D & - & 1 & - & - \\
MAC-A & - & 2 & - & - \\
MAC-I & - & 2 & - & - \\
Msc-A & - & - & 1 & - \\
Msi-A & 1 & - & - & - \\
MTC-A & 1 & - & - & - \\
Total & 22 & 13 & 4 & 7 \\
\hline
\end{tabular}

*Two isolates were $M$. avium subsp. paratuberculosis.

patients. Unfortunately, since the $M$. simiae and $M$. terrae isolates were obtained through a diagnostic laboratory, it was not possible to analyse if these isolates were clinically significant.

The animal isolates were found to belong exclusively to the Mav sequevars. In contrast to the human isolates, Mav-A was the most common sequevar. Both $M$. avium subsp. paratuberculosis isolates had the Mav-A sequevar, while the two Mav-B isolates were derived from pigs.

\section{Phylogenetic analysis}

A phylogenetic tree was constructed using the UPGMA algorithm with all the sequevars described here, all previously described Mav, MAC and Msc sequevars, as well as the Mtb-A sequevar and the published $M$. babana and $M$. paratuberculosis ITS sequences (Frothingham \& Wilson, 1994; Ji et al., 1994; Van der Giessen et al., 1994) (Fig. 2). All Mav, Min and MAC sequevars fell into one group containing three clusters. This group was separate from the M. scrofulaceum sequevars Msc-A and Msc-B, confirming the phylogenetic identity of the MAI complex.

The MAC-A sequevar clustered with the Mav sequevars, despite being identified as $M$. intracellulare by sequencing part of the 16S rRNA gene and Accuprobe analysis. However, it is noteworthy that Frothingham \& Wilson $(1993,1994)$ obtained a similar clustering using a different algorithm.

\section{DISCUSSION}

The current routine of clinical laboratory testing is mostly limited to the identification of organisms within the MAI complex, but without differentiation between $M$. avium

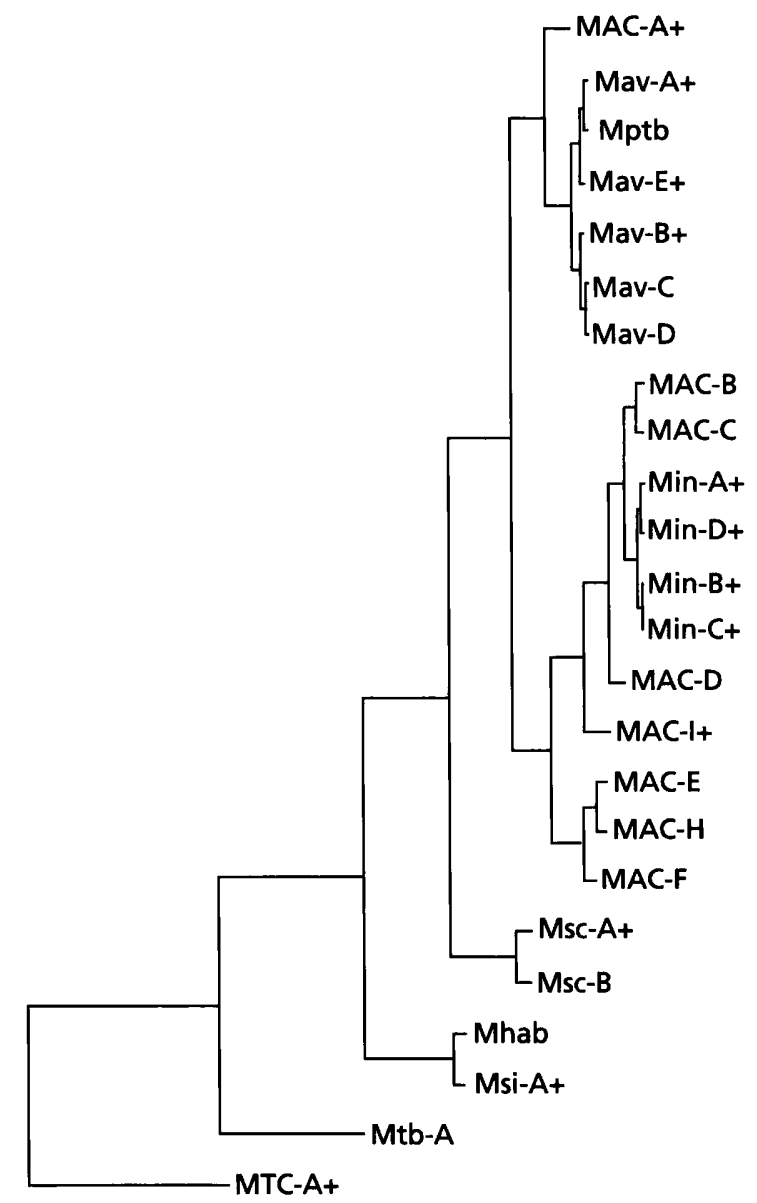

Fig. 2. Phylogenetic tree showing all ITS sequences mentioned in the text. A ' + ' indicates ITS sequences reported found in clinical isolates. Mhab stands for $M$. habana (Ji et al., 1994), Mptb for M. paratuberculosis (van der Giessen et al., 1994) and Mtb for M. tuberculosis complex (Frothingham et al., 1994).

and M. intracellulare (Inderlied et al., 1993). However, the potential clinical importance of species identification has been suggested by reports that these two species have different drug susceptibilities (Guthertz et al., 1989; Saito et al., 1990) and different prognosis in pulmonary disease (Yamori \& Tsukamura, 1992), and that MAI complex isolates from AIDS patients are almost exclusively $M$. avium (Frothingham \& Wilson, 1994; Tsang et al., 1992).

Routine differentiation between $M$. avium and $M$. intracellulare organisms has not been possible by biochemical methods and is elaborate as well as not wholly accurate by serological typing (De Smet et al., 1995; Saito et al., 1990; Tsang et al., 1992; Wayne et al., 1993). Using commercial kits based on species-specific oligonucleotides (the Accuprobe kits, Gen-Probe), $M$. tuberculosis complex, $M$. avium, $M$. intracellulare, $M$. gordonae and $M$. kansasii can be identified from cultures in a few hours. With the development of PCR and the direct sequencing of PCR products, it has become feasible to quickly identify any mycobacterial isolate using a single test by sequencing part of the 16S rRNA gene (Böddinghaus et al., 1990; Kirschner et al., 1993). However, these DNA-based tests 
do not show variations within these species and therefore are not suitable for strictly epidemiological investigations.

'ITS sequevar' typing, based on sequencing of the internal transcribed spacer (ITS) between the 16S and 23S rRNA genes, has recently been described to subdivide the MAI complex (Frothingham \& Wilson, 1993). By analysing 46 isolates from different clinical sources, we found that 22 out of $25 \mathrm{M}$. avium isolates from human sources in the UK belong to the same sequevar, Mav-B, in both AIDS patients and child lymphadenitis. On the other hand, both Mav-A and Mav-B were common among the animal-derived strains. Among the isolates from 12 elderly patients with pulmonary disease, we identified eight different sequevars.

It is unlikely that the sequence differences between the different isolates are due to errors in the PCR. It is well established that the Taq polymerase often incorporates the wrong nucleotide, which can lead to incorrect sequence data if this aberrant fragment is cloned before sequencing (Eckert \& Kunkel, 1993). However, this is unlikely to be the explanation for the sequence variation we found. Because we started with a large amount of DNA (over $10 \mathrm{ng}$ or $10^{6}$ copies) and sequenced the whole pool of PCR products, mistakes in the amplification of single nucleotides in a single copy would not affect the direct sequencing data of the whole pool. The different sequences thus truly represent genetic diversity or different 'sequevars'.

\section{Sequevars within species}

This study extends the number of sequevars within the $M$. avium species to five (Mav-A to $-\mathrm{E}$ ). The finding of the Mav-A sequevar for both $M$. avium subsp. paratuberculosis isolates tested supports the classification of this mycobacterium as a subspecies of M. avium (Thorel et al., 1990). However, in another study the ITS of $M$. avium subsp. paratuberculosis contained one basepair difference from the Mav-A and two basepairs from Mav-B ITS sequences (Van der Giessen et al., 1994), indicating the existence of at least two sequevars in this subspecies. Similarly, a recent publication has described the ITS sequence of $M$. babana, which is an older name for M. simiae (Ji et al., 1994). There were seven basepair differences between this published sequence and our Msi-A sequevar sequence, suggesting heterogeneity within this species as well. On the other hand, no ITS sequence variation was found among isolates of the $M$. tuberculosis complex from different clinical sources (Frothingham et al., 1994; Glennon et al., 1994). Also, single-strand conformation polymorphism (SSCP) typing of the ITS region from 75 $M$. leprae samples indicated that there was no variation in the ITS of this species (De Wit \& Klatser, 1994).

ITS sequence variation in $M$. intracellulare had not been reported before, but it corresponds well with our previous data showing considerable RFLP variation with the probe pMB22 in this species (De Smet et al., 1995). The two MAC sequevars were confirmed as belonging to the MAI complex by sequencing part of their $16 \mathrm{~S}$ rRNA genes, with the MAC-A sequevar isolates having an identical $16 \mathrm{~S}$
rDNA sequence to $M$. intracellulare, while the MAC-I sequence corresponded to ' $M$. intracellulare serovar 18' (Böddinghaus et al., 1990). This underlines the higher variability of the ITS sequences compared to $16 \mathrm{~S}$ rDNA sequences. While $16 \mathrm{~S}$ rDNA sequences cannot subdivide $M$. avium and can divide $M$. intracellulare into only two groups, sequevar typing divides the MAI complex into at least 16 sequevars (Mav-A to Mav-E, Min-A to Min-D, and MAC-A to MAC-I), which may be useful for future epidemiological studies of MAI complex infection.

It remains possible that several other sequevars have not been identified to date. To maintain a consistent naming of newly discovered ITS sequevars, we propose that either the corresponding author and/ or Dr R. Frothingham (rfr@galactose.mc.duke.edu) (Frothingham \& Wilson 1993, 1994) should be contacted to assign names.

\section{Comparison of sequevar analysis with other techniques for species identification}

All isolates were included in this study after they had been identified as belonging to the MAI complex by biochemical analysis in a diagnostic laboratory. Sequence analysis revealed that three of these were $M$. scrofulaceum, $M$. simiae and $M$. terrae and had thus initially been incorrectly identified. Frothingham \& Wilson (1994) also found that two isolates, originally identified as $M$. avium, were $M$. celatum as determined by DNA sequencing. Thus, typing by sequencing either the $16 \mathrm{~S}$ rDNA or the ITS sequences may be a more accurate way of identifying mycobacterial species than microbiological and biochemical techniques.

The Accuprobe kit is based on hybridization of a speciesspecific oligonucleotide on the $16 \mathrm{~S}$ rRNA. There was perfect agreement between ITS sequevar typing and Accuprobe species identification. Interestingly, the MACA isolate was identified as $M$. intracellulare by both the $16 \mathrm{~S}$ rDNA sequencing and the Accuprobe analysis, although its ITS sequence was more closely related to the Mav sequevar sequences in the phylogenetic analysis. All of the MAI complex isolates tested with the Accuprobe could be classified as $M$. avium or $M$. intracellulare in this study. But it has to be pointed out that strains of the MAC-B, MAC$C$ and MAC-F sequevars have been reported to be negative with the closely related Genprobes (radiolabelled versions of the Accuprobes), while a MAC-D isolate was positive with both Genprobes (Frothingham \& Wilson 1994).

Serotyping has been used for species identification within the MAI complex, with serovars $1-6,8-11,21$ and 28 considered to be $M$. avium, while those with serovars 7 , 12-20 and 25 are assigned to $M$. intracellulare (Saito et al., 1989; Böddinghaus et al., 1990; Tomioka et al., 1993). For the isolates belonging to the Mav or Min sequevars, there was a good, but not perfect correlation between the serotyping and the ITS sequevar results for species assignation, with only two exceptions out of 37 isolates. Three of the four MAC isolates were of serovars usually associated with $M$. intracellulare. The three isolates identi- 
fied by $16 \mathrm{~S} \mathrm{rDNA}$ sequencing as $M$. scrofulaceum, $M$. simiae and $M$. terrae were originally incorrectly identified as belonging to the MAI complex and had thus also been serotyped (De Smet et al., 1995). Interestingly, the $M$. simiae and $M$. terrae isolates had serotypes associated with the MAI complex, while the $M$. scrofulaceum isolate could not be typed.

Taking these findings together, it has to be concluded that serovars are not entirely species specific, a conclusion that is supported by other anecdotal reports of similar discrepancies between serotyping and DNA-based techniques within the MAI complex (Frothingham \& Wilson, 1993; Wayne et al., 1993).

\section{Association of sequevar with clinical source}

Our data suggest that Mav-B is associated with human disease, while Mav-A is found predominantly in animal infections. Frothingham \& Wilson (1994) also found mainly Mav-B among isolates from AIDS patients. A genetic difference between $M$. avium infecting humans and those infecting animals has been demonstrated previously: the insertion sequence IS901 is common among animal isolates, but rare among isolates from human or environmental sources (Kunze et al., 1992). There is considerable variation in the virulence of $M$. avium isolates in several experimental systems. This is often correlated with the difference in colony morphology or the serovar, reflecting differences in the cell wall, although this remains controversial (Wayne \& Kubica, 1986; Tsang et al., 1992; Belisle \& Brennan, 1994; Pedrosa et al. 1994). Pedrosa et al. (1994) recently demonstrated that most $M$. avium isolates from animals were more virulent for mice than those from AIDS patients. This virulence for mice correlated well with RFLP typing using IS901. It is therefore plausible that different genetic subgroups of the MAI complex have different virulence characteristics or host range. Thus for as yet unknown virulence or hostrange factors, the Mav-B subgroup is more likely to infect humans. Different animal species may also be susceptible to different strains of $M$. avium, since both pig isolates were Mav-B while the cow and pigeon isolates were MavA.

Alternatively, it is possible that Mav-B is the most common sequevar in the environment, which is reflected in the infection rate of AIDS patients. The prevalence of serovars in AIDS patients was correlated with those found among environmental isolates in several cities in the USA (Tsang et al., 1992). However, our cow and pigeon isolates were all Mav-A (three out of three isolates), supporting the hypothesis that there is a correlation between sequevar and host range. It should also be noted that most $M$. avium serovar reference strains, which were mainly derived from animal and environmental sources, belonged to sequevar Mav-A (Frothingham \& Wilson, 1993).

On the other hand, there was no association between sequevars and host among the human pulmonary disease isolates. While the majority belonged to $M$. intracellulare
(Min and MAC sequevars), there were also two infections due to $M$. avium. This suggests that all these different sequevars may have similar virulence factors for causing pulmonary infections.

\section{Concluding remarks}

Our findings suggest that individuals with systemic immune deficiency (e.g. AIDS) or infants with an immature immune system harbour predominantly $M$. avium sequevar Mav-B, while chronic infections with $M$. intracellulare of different Min and MAC sequevars are established in elderly patients. Organisms from the MAI complex cause a wide range of pathogenic conditions not only in humans but also in a wide range of hosts. It would therefore be of interest to analyse more animal and environmental isolates to further investigate whether individual sequevars associate with distinct pathogenic mechanisms or hosts and to characterize the virulence factors involved.

\section{ACKNOWLEDGEMENTS}

We thank Dr P. A. Jenkins, Dr R. A. Ollar and Dr A. Nolan for supplying MAI complex isolates. We are grateful to Adrian Hills and Brian Robertson for help with the computer analysis of DNA sequences.

\section{REFERENCES}

Belisle, J. T. \& Brennan, P. J. (1994). Molecular basis of colony morphology in Mycobacterium avium. Res Microbiol 145, 237-242.

Boddinghaus, B., Wolters, J., Heikens, W. \& Bbttger, E. C. (1990). Phylogenetic analysis and identification of different serovars of Mycobacterium intracellulare at the molecular level. FEMS Microbiol Lett 70, 197-204.

Chiodini, R. J. (1989). Crohn's disease and the mycobacterioses: a review and comparison of two disease entities. Clin Microbiol Rev 2, 90-117.

De Smet, K. A. L., Hellyer, T. J., Khan, A. W., Brown, I. N. \& Ivanyi, J. (1995). Genetic and serovar typing of clinical isolates of the Mycobacterium avium-intracellulare complex. Tubercle Lung Dis (in press).

De Wit, M. Y. L. \& Klatser, P. R. (1994). Mycobacterium leprae isolated from different sources have identical sequences of the spacer region between $16 \mathrm{~S}$ and $23 \mathrm{~S}$ ribosomal RNA genes. Microbiology 140, 1983-1987.

Eckert, K. A. \& Kunkel, T. A. (1993). The fidelity of DNA polymerases used in the polymerase chain reaction. In PCR: $a$ Practical Approach, pp. 225-244. Edited by M. J. McPherson, P. Quircke \& G. R. Taylor. Oxford: IRL Press.

Frothingham, R. \& Wilson, K. H. (1993). Sequence-based differentiation of strains in the Mycobacterium avium complex. J Bacteriol 175, 2818-2825.

Frothingham, R. \& Wilson, K. H. (1994). Molecular phylogeny of the Mycobacterium avium complex demonstrates clinically meaningful divisions. J Infect Dis 169, 305-312.

Frothingham, R., Hills, H. G. \& Wilson, K. H. (1994). Extensive DNA sequence conservation throughout the Mycobacterium tuberculosis complex. J Clin Microbiol 32, 1639-1643.

Glennon, M., Smith, T., Cormican, M., Noone, D., Barry, T., Maher, M., Dawson, M., Gilmartin, J. J. \& Gannon, F. (1994). The 
ribosomal intergenic spacer region: a target for the PCR based dianosi of tuberculosis. Tubercle Lung Dis 75, 353-360.

Good, R. C. \& Beam, R. E. (1984). Seroagglutination. In The Mycobacteria: a Source Book, Part A, pp. 105-122. Edited by G. P. Kubica \& L. G. Wayne. New York: Marcel Dekker.

Guthertz, L. S., Damsker, B., Bottone, E. J., Ford, E. G., Midura, T. F. \& Janda, M. (1989). Mycobacterium avium and Mycobacterium intracellulare infections in patients with and without AIDS. $J$ Infect Dis 160, 1037-1041.

Hellyer, T. J., Brown, I. N., Dale, J. W. \& Easmon, C. S. F. (1991). Plasmid analysis of Mycobacterium avium-intracellulare (MAI) isolated in the United Kingdom from patients with and without AIDS. $J$ Med Microbiol 34, 225-231.

Hellyer, T. J., Brown, I. N., Taylor, M. B., Allen, B. W. \& Easmon, C. S. F. (1993). Gastro-intestinal involvement in Mycobacterium aviumintracellulare infection of patients with HIV. J Infect 26, 55-66.

Inderlied, C. B., Kemper, C. A. \& Bermudez, L. E. M. (1993). The Mycobacterium avium complex. Clin Microbiol Rev 6, 266-310.

Ji, Y. E., Kempsell, K. E., Colston, M. J. \& Cox, R. A. (1994). Nucleotide sequences of the spacer-1, spacer-2 and trailer regions of the $r r n$ operons and secondary structures of precursor $23 \mathrm{~S}$ rRNAs and precursor $5 \mathrm{~S}$ rRNAs of slow-growing mycobacteria. Microbiology 140, 1763-1773.

Kirschner, P., Springer, B., Vogel, U., Meier, A., Wrede, A., Kiekenbeck, M., Bange, F.-C. \& Bbttger, E. C. (1993). Genotypic identification of mycobacteria by nucleic acid sequence determination: report of a 2 -year experience in a clinical laboratory. $J$ Clin Microbiol 31, 2882-2889.

Kunze, Z. M., Portaels, F. \& McFadden, J. J. (1992). Biologically distinct subtypes of Mycobacterium avium differ in possession of insertion sequence IS901. J Clin Microbiol 30, 2366-2372.

Kwok, S. \& Higuchi, R. (1989). Avoiding false positives with PCR. Nature 339, 237-238.

Lai, K. K., Stottmeier, K. D., Sherman, I. H. \& McCabe, W. R. (1984). Mycobacterial cervical lymphadenopathy, relation of etiolgical agents to age. $J$ Am Med Ass 251, 1286-1288.

Pedrosa, J., Flórido, M., Kunze, Z. M., Castro, A. G., Portaels, F., McFadden, J., Silva, M. T. \& Appelberg, R. (1994). Characterization of the virulence of Mycobacterium avium complex (MAC) isolates in mice. Clin Exp Immunol 98, 210-216.

Rosenzweig, D. Y \& Schlueter, D. P. (1981). Spectrum of clinical disease in pulmonary infection with Mycobacterium aviumintracellulare. Rev Infect Dis 3, 1046-1051.

Saito, H., Tomioka, H., Sato, K., Tasaka, H., Tsukamura, M., Kuze, F. \& Asano, K. (1989). Identification and partial characterisation of Mycobacterium avium and Mycobacterium intracellulare by using DNA probes. J Clin Microbiol 27, 994-997.
Saito, H., Tomioka, H., Sato, K., Tasaka, H. \& Dawson, D. J. (1990). Identification of various serovar strains of Mycobacterium avium complex by using DNA probes specific for Mycobacterium avium and Mycobacterium intracellulare. J Clin Microbiol 28, 1694-1697.

Thoen, C. O. (1994). Mycobacterium avium infections in animals. Res Microbiol 145,173-176.

Thorel, M.-F., Krichevsky, M. \& Lévy-Frébault, V. (1990). Numerical taxonomy of mycobactin-dependent mycobacteria, amended description of Mycobacterium avium, and description of Mycobacterium avium subsp. avium subsp. nov., Mycobacterium avium subsp. paratuberculosis subsp. nov. and Mycobacterium avium subsp. silvaticum subsp. nov. Int J Syst Bacteriol 40, 254-260.

Tomioka, H., Saito, H., Sato, K., Tasaka, H. \& Dawson, D. J. (1993). Identification of Mycobacterium avium complex strains belonging to serovars $21-28$ by three commercial DNA probes tests. Tubercle Lung Dis 74, 91-95.

Tsang, A. Y., Denner, J. C., Brennan, P. J. \& McClatchy, J. K. (1992). Clinical and epidemiological importance of typing of Mycobacterium avium complex isolates. J Clin Microbiol 30, 479-484.

Van der Giessen, J. W. B., Haring, R. M. \& Van der Zeijst, B. A. M. (1994). Comparison of $23 S$ ribosomal RNA genes and the spacer region between the $16 \mathrm{~S}$ and $23 \mathrm{~S}$ rRNA genes of the closely related Mycobacterium avium and Mycobacterium paratuberculosis and the fastgrowing Mycobacterium phlei. Microbiology 140, 1103-1108.

Van Soolingen, D., Hermans, P. W. M., De Haas, P. E. W., Soll, D. R. \& Van Embden, J. D. A. (1991). Occurrence and stability of insertion sequences in Mycobacterium tuberculosis complex strains: evaluation of an insertion sequence-dependent DNA polymorphism as a tool in the epidemiology of tuberculosis. $J$ Clin Microbiol 29, 2578-2586.

Wayne, L. G. \& Kubica, G. P. (1986). The mycobacteria. In Bergey's Manual of Systematic Bacteriology, vol. 2, pp. 1435-1457. Edited by J. G. Holt, P. H. A. Sneath, N. S. Mair \& M. E. Sharpe. Baltimore: Williams \& Wilkins.

Wayne, L. G., Good, R. C., Tsang, A., Butler, R., Dawson, D., Groothuis, D., Gross, W., Hawkins, J., Kilburn, J., Kubin, M., Schröder, K. H., Silcox, V. A., Smith, C., Thorel, M.-F., Woodley, C. \& Yakrus, M. A. (1993). Serovar determination and molecular taxonomic correlation in Mycobacterium avium, Mycobacterium intracellulare and Mycobacterium scrofulaceum: a cooperative study of the International Working Group on Mycobacterial Taxonomy. Int J Syst Bacteriol 43, 482-489.

Yamori, S. \& Tsukamura, M. (1992). Comparison of prognosis for pulmonary diseases caused by Mycobacterium avium and by Mycobacterium intracellulare. Chest 102, 89-90.

Received 12 April 1995; revised 23 June 1995; accepted 28 June 1995. 